VS18-02

\section{High-pressure studies on magnetocaloric materials}

Karen Friese ${ }^{1}$, Andreas Eich², Paul Hering ${ }^{1}$, Martin Meven ${ }^{3}$, Andrzej Grzechnik $^{2}$

1. Jülich Centre for Neutron Science-2, Forschungszentrum Jülich GmbH, Jülich, Germany

2. Institute for Crystallography, RWTH Aachen University, Aachen, Germany

3. Institute of Crystallography, RWTH Aachen University and Jülich Centre for Neutron Science, Forschungszentrum Jülich $\mathrm{GmbH}$ at Heinz Maier-Leibnitz Zentrum (MLZ), Garching, Germany

email: k.friese@fz-juelich.de

The magnetocaloric effect describes the change of the adiabatic temperature of a material with the application of a magnetic field. This effect forms the basis of magnetic refrigeration technologies that could potentially replace conventional vapor compression cooling. Individual compounds in the system $\mathrm{Mn}_{5-\mathrm{x}} \mathrm{Fe}_{\mathrm{x}} \mathrm{Si}_{3}$ (all of them hexagonal at ambient conditions) undergo a variety of magnetic phase transitions as a function of temperature and composition which are accompanied by a significant (for some compounds inverse) magnetocaloric effect $[1,2]$. The magnetic transitions are also clearly reflected in changes of the crystal lattice [2]. The application of hydrostatic pressure changes the interatomic distances in the crystal structure and strongly influences the magnetic exchange interactions. Pressure experiments can therefore provide crucial information on magnetocaloric materials and help to understand the fundamental mechanism of the magnetocaloric effect. We performed in situ temperature dependent powder diffraction experiments at high pressures at synchrotron sources (Petra III and ESRF), as well as in situ high pressure single crystal diffraction experiments at laboratory sources and complementary magnetization measurements under high pressure. In addition, we are currently developing the experimental setup for high pressure neutron diffraction experiments using thermal neutrons at the single crystal diffractometer on the hot source (HEiDi) at the MLZ in Garching where first results show that the diffraction data collected at HEIDI can be used for full and reliable structure refinements [3]. Our experiments on the magnetocaloric compounds show that the magnetic properties are strongly influenced by the application of hydrostatic pressure. The effect of pressure on the unit cell volume is similar to the effect of chemical substitution (or "chemical pressure"), as the substitution of $\mathrm{Mn}$ by the smaller Fe in the series $\mathrm{Mn}_{5-\mathrm{x}} \mathrm{Fe}_{\mathrm{x}} \mathrm{Si}_{3}$ also leads to a reduction of the unit cell volume. A detailed comparison of hydrostatic and "chemical pressure" will be provided. On the other hand, a detailed comparison of the influence of pressure and temperature on the studied materials surprisingly shows that the degree of distortion of the (hexagonal) unit cell is far more sensitive to temperature than to pressure - a fact that is probably closely related to the magnetocaloric properties of the materials.
References:

[1] Songlin, et al., J. Alloys Comp. 334, 249-252 (2002)

[2] P. Hering, K. Friese, J. Voigt et.al., Chem. Mat. 2015, 27, 7128-

$7136(2015)$

[3] A. Grzechnik, M. Meven, K. Friese, J. Appl. Cryst. 51, 351-356 (2018)

Keywords: High pressure, single crystal diffraction, magnetocaloric materials 